\title{
Patterns of cyclin A and B1 immunostaining in papillary thyroid carcinoma
}

\author{
Anna Cyniak-Magierska', Magdalena Stasiak', Maciej Naze², Marek Dedecjus², Jan Brzeziński², \\ Andrzej Lewiński ${ }^{1}$ \\ ${ }^{1}$ Department of Endocrinology and Metabolic Diseases, Polish Mother's Memorial Hospital - Research Institute, Lodz, \\ Poland \\ 2 Department of General, Oncological and Endocrine Surgery, Polish Mother's Memorial Hospital - Research Institute, \\ Lodz, Poland
}

Cyniak-Magierska A, Stasiak M, Naze M, Dedecjus M, Brzeziński J, Lewiński A. Patterns of cyclin A and B1 immunostaining in papillary thyroid carcinoma. Ann Agric Environ Med. 2015; 22(4): 741-746. doi: 10.5604/12321966.1185787

\begin{abstract}
Introduction and objectives. Cyclin A, encoded by CCNA (cyclin A) gene with locus in chromosome 4q27, and cyclin B1, encoded by CCNB1 (cyclin B1) gene with locus in chromosome 5q12, are proteins that play a key role in the passage through the restriction point in $\mathrm{G} 2$ phase of the cell cycle. The aim of the study was to analyse immunohistochemically the expression of cyclins A and B1 in different variants of papillary thyroid carcinoma (PTC).

Material and methods. The immunostaining patterns of the proteins in question in the tissue of 40 resected PTC ( 20 cases of classic variant of PTC, 9 cases of PTC follicular variant and 11 cases of other non-classic variants of PTC) were investigated. Results. On analyzing cyclin A and B1 expression, positive staining in $90 \%$ cases of PTC were observed. The study revealed a significant difference in expression of cyclins A and B1 between classic and non-classic variants of PTC. The expression of both examined cyclins was weaker in the classic variant of PTC. In the group of follicular variant of PTC, the expression of cyclins was of medium intensity and in the group of other non-classic variants of PTC, the expression was clearly higher. Conclusions. The results of the presented study suggest that cyclins A and B1 expression may have a characteristic pattern of immunostaining for particular variants of PTC. If the obtained results are confirmed in a larger group of patients, the diagnostic panel constructed of the antibodies against these proteins may increase the diagnostic accuracy in PTC cases.
\end{abstract}

\section{Key words}

Cyclin A, cyclin B1, papillary thyroid carcinoma, immunohistochemistry

\section{INTRODUCTION}

Papillary thyroid carcinoma (PTC) is the most common of all thyroid cancers and the incidence of this neoplasm has been increasing over the last decade. Its prognosis is associated with patient's age, tumour size, as well as histological parameters, including extracapsular invasion, extrathyroidal extension, lymph node and distant metastasis and histological variants. These histological variants, which include the classic variant of PTC, follicular variant of PTC and other variants, such as the tall-cell variant, the columnar-cell variant or the variant with diffuse sclerosis, are related to tumour aggressiveness and metastatic potential. In addition, in PTC, several molecular changes have been typically described (e,g, RET/ $P T C, N T R K 1$ rearrangements and/or sporadic mutations of $B R A F$ or $R A S$ oncogene) $[1,2,3]$.

Thyroid nodules are common ultrasound findings, detected in approximately $19-67 \%$ of the general adult population. The aim of further approach is to differentiate between benign nodule and malignancy. Ultrasoundguided fine needle aspiration biopsy (FNAB) is the most important tool for this purpose, although its accuracy is not always sufficient due to a relatively high incidence of indeterminate or suspicious cytologic features. Most of these patients undergo diagnostic thyroidectomy, which

address for correspondence: Andrzej Lewiński, Department of Endocrinology and Metabolic Diseases, Polish Mother's Memorial Hospital - Research Institute, Rzgowska 281/289, 93-338 Lodz, Poland

E-mail: alewin@csk.umed.lodz.pl

Received: 15 January 2014; accepted: 13 May 2014 frequently reveals a benign lesion. Thus, many novel potential markers are studied to facilitate the diagnosis of malignancy and to determine tumour type and progression. Several biomarkers which might be useful adjuncts in the assessment of proliferation and apoptosis in PTC have been identified. Dysregulation of the normal cell cycle machinery, leading to unrestrained cell proliferation, is integral to the neoplastic process, and the loss of regulatory control of the cell cycle is a hallmark of cancer. Cyclins, the regulatory subunits of cyclin-dependent kinases (CDK), control the passage of proliferating cells through key checkpoints in the cell cycle. The overexpression of positive cell regulators (i.e. cyclins) may overwhelm the arrest mechanism of the normal cell cycle and lead to uncontrolled cell proliferation. An important checkpoint in the cell cycle progression is at G2/M boundary [4]. G2/M transition is regulated by the complex of CDK I and two cyclins: cyclin A, encoded by CCNA (cyclin A) gene with locus in chromosome 4q27 and cyclin B1, encoded by CCNB1 (cyclin B1) gene with locus in chromosome $5 \mathrm{q} 12$. Cyclin A activates CDK to regulate proliferation and cell cycle progression through the S phase to the G2/M checkpoint [5]. Cyclin B1 works selectively for G2/M transition.

Cyclin A and cyclin B1 expressions have possible prognostic value in breast, hepatocellular, squamous oesophageal cancers and in non-Hodgkin's lymphoma $[6,7,8]$; however, their role in PTC has been less studied.

Objectives. The aim of the study was to analyse immunohistochemically the expression of cyclins A and B1 in different variants of PTC. The diagnostic utility of such 
immunostaining patterns in differentiation of particular variants of PTC was also evaluated.

\section{MATERIALS AND METHOD}

The procedures, used in the study were approved by the Ethical Committee of the Medical University of Lodz, Poland.

Tissue specimens. A total of 40 PTC cases were selected from the surgical pathology files of the hospital, comprising 20 cases of classic variant of PTC (mean age of the studied patients: 45; SD 12.3 years; range: 19 - 82 years) and 20 cases of other non-classic variants of PTC (mean age of the studied patients: 43.5 ; SD 17.1 years; range: $24-69$ years). The patients were diagnosed and operated on between $2002-2004$ at the Department of Endocrine and General Surgery, Institute of Endocrinology, Medical University of Lodz. The experimental protocol included only females. All patients diagnosed with thyroid cancer before or at the time of surgery underwent total thyroidectomy and regional lymphadenectomy. The remaining patients, diagnosed with cancer during routine histopathologic examinations, were re-operated, the operation was radicalized and regional lymphadenectomy was performed. Following the approved procedures, all patients received post-operative radioactive iodine therapy, followed by L-T $\mathrm{T}_{4}$ suppression treatment. All the clinical charts and histopathology reports were reviewed for data regarding the patient's age, tumour size and the presence of lymph node metastasis. Histopathological diagnoses for carcinomas, according to the WHO Classification of Tumours [9], were obtained from pathomorphological reports (Tab.1), together with TNM classification and AJCC stage groupings [10].

Two pathologists reviewed all the cases to confirm the obtained diagnoses. At that review of $\mathrm{H} \& \mathrm{E}$-stained sections, paraffin blocks were selected for immunohistochemistry to include both the representative sections of the analyzed tumours and the rims of morphologically normal thyroid tissue around them, the latter serving as comparative tissue.

Immunohistochemistry. Archival paraffin-embedded tumour tissue was analyzed by immunohistochemistry for cyclin A and B1 expressions. Formalin-fixed paraffinembedded tissue sections ( $4 \mu \mathrm{m}$ thickness) were dewaxed in xylene and rehydrated through graded alcohols to water. Antigen retrieval was performed in citrate buffer $(\mathrm{pH}$ 6.0; Dako, Glostrub, Denmark) inside a microwave pressure cooker.

Primary antibody characteristics, the conditions of incubation and the manufacturer's name for each of the cyclins are presented in Table 2. After incubation, the slides were washed in Tris Buffered Saline (TBS), after which the incubations were carried out using an En Vision/HRP/DAB+ kit (Dako). The slides were counterstained in haematoxylin and mounted on Faramount (Dako). For negative control, TBS replaced the primary antibody.

Evaluation of immunostaining. Immunostained tissue sections were evaluated by estimating the percentage of tumour cells staining positive with monoclonal antibody without any knowledge of pathologic diagnosis. For the evaluation of positivity, the positively stained nuclei and cytoplasm (for cyclin A and cyclin B1, respectively) were
Table 1. Age, histopathological diagnosis, TNM classification and American Joint Committee on Cancer (AJCC) stage groupings of malignant thyroid tumours in studied patients

\begin{tabular}{|c|c|c|c|c|}
\hline Case No. & Age & $\begin{array}{c}\text { Histopathological } \\
\text { variant of PTC }\end{array}$ & $\begin{array}{l}\text { TNM staging } \\
\text { system }\end{array}$ & $\begin{array}{l}\text { AJCC grouping } \\
\text { system }\end{array}$ \\
\hline 1 & 48 & classic & $\mathrm{T} 2 \mathrm{~N} \times \mathrm{MO} 0$ & ॥ \\
\hline 2 & 25 & classic & T2NOMO & I \\
\hline 3 & 31 & classic & T4N1M0 & I \\
\hline 4 & 70 & classic & T2NOMO & II \\
\hline 5 & 30 & classic & T2NOMO & I \\
\hline 6 & 60 & classic & T3N1Mx & III \\
\hline 7 & 24 & classic & T1aNoMo & 1 \\
\hline 8 & 51 & classic & T1bNOMO & 1 \\
\hline 9 & 19 & classic & T4N1Mx & 1 \\
\hline 10 & 49 & classic & $\mathrm{T} 2 \mathrm{NxMx}$ & ॥ \\
\hline 11 & 22 & classic & $\mathrm{T} 2 \mathrm{~N} 1 \mathrm{Mx}$ & I \\
\hline 12 & 82 & classic & T2NxMx & II \\
\hline 13 & 61 & classic & T3NOMO & III \\
\hline 14 & 37 & classic & T2N1Mx & I \\
\hline 15 & 49 & classic & T1aN0Mx & I \\
\hline 16 & 64 & classic & T1aN0Mx & 1 \\
\hline 17 & 52 & classic & T1bNOMO & 1 \\
\hline 18 & 58 & classic & T1aN1Mx & III \\
\hline 19 & 35 & classic & $\mathrm{T} 2 \mathrm{NxMx}$ & 1 \\
\hline 20 & 42 & classic & T1aN1Mx & I \\
\hline 21 & 50 & follicular & T1bN0M0 & 1 \\
\hline 22 & 24 & follicular & $\mathrm{T} 2 \mathrm{~N} 1 \mathrm{M} 0$ & 1 \\
\hline 23 & 35 & follicular & T1aNoMo & I \\
\hline 24 & 42 & follicular & T2NOMO & 1 \\
\hline 25 & 43 & follicular & T1aN1M0 & I \\
\hline 26 & 30 & follicular & T1bNOMO & I \\
\hline 27 & 69 & follicular & T3N1Mx & III \\
\hline 28 & 48 & follicular & T1aNoMo & 1 \\
\hline 29 & 41 & follicular & $\mathrm{T} 2 \mathrm{~N} 1 \mathrm{M} 0$ & 1 \\
\hline 30 & 38 & tall-cell & T1bNOM0 & I \\
\hline 31 & 37 & tall-cell & T1aN1Mx & I \\
\hline 32 & 47 & tall-cell & T1bN1Mx & III \\
\hline 33 & 66 & tall-cell & T1bNOM0 & 1 \\
\hline 34 & 47 & tall-cell & T2NxMx & II \\
\hline 35 & 52 & tall-cell & T1bN0M0 & I \\
\hline 36 & 42 & tall-cell & $\mathrm{T} 2 \mathrm{~N} 1 \mathrm{M} 0$ & I \\
\hline 37 & 62 & columnar-cell & T3N1Mx & III \\
\hline 38 & 30 & columnar-cell & T1aNoMo & I \\
\hline 39 & 24 & columnar-cell & T1aNxMx & 1 \\
\hline 40 & 43 & $\begin{array}{l}\text { with diffuse } \\
\text { sclerosis }\end{array}$ & T3N1Mx & I \\
\hline
\end{tabular}

counted. The nuclear staining in the normal thyroid tissue surrounding PTC, was also evaluated at the same time as the tumour, serving as an internal control.

Staining index. All the stained sections were examined on an Olympus microscope (Olympus CHS, Olympus, Tokyo, Japan), using an eyepiece graticule (PZO, Warsaw, Poland) to facilitate cell counting, at a higher magnification (400 
Table 2. Characteristics of antibodies used and incubation conditions

\begin{tabular}{lll}
\hline & \multicolumn{1}{c}{ Cyclin A } & \multicolumn{1}{c}{ Cyclin B1 } \\
\hline Antibody/animal & Rabbit & Mouse \\
\hline Antibody/isotype & IgG polyclonal & lgG 1 monoclonal \\
\hline Antibody/clone & $\mathrm{H}-432 ;$ sc-751 & GNS1; sc-245 \\
\hline Dilution & $1: 100$ & $1: 100$ \\
\hline Incubation & Whole night $\left(4^{\circ} \mathrm{C}\right)$ & Whole night $\left(4^{\circ} \mathrm{C}\right)$ \\
\hline Manufacturer & Santa Cruz & Santa Cruz \\
& Biotechnology & Biotechnology \\
\hline
\end{tabular}

$\mathrm{x})$ at which a minimum of 1,000 cells were counted in the area with positive staining. The percentage of tumour cells stained with the antibody was regarded as the staining index. Staining results were defined as negative (expression $<5 \%$ ) or positive. A score (1-3) was used to classify positive cells (1: between $5-10 \%$ of positive cells; 2 : between $10-50 \%$ of positive cells; $3:>50 \%$ of positive cells).

Statistical analyses. The multivariate variance analyses (Fischer-Snedecor test) was used to assess whether there was a difference in the expression of the antigens in question between PTC of different variants. Statistical significance was determined at the level of $\mathrm{p}<0.05$.

Statistica for Windows 7.0 was applied for calculations.

\section{RESULTS}

Cyclin A expression. On analyzing the cyclin A expression, positive staining was observed in $90 \%$ of the cases $(n=36 / 40)$. The staining signal of cyclin A was observed predominantly in the nuclei in $27 \%(\mathrm{n}=11)$ of cases, in cytoplasm in $40 \%(16)$, and both in nuclei and cytoplasm in $23 \%(n=9)$ of the cases.

In the group of classic variant of PTC, positive staining was observed in $80 \%$ of the cases $(n=16 / 20)$. The staining signal of cyclin A was observed predominantly in the nuclei in 35\% $(n=7)$ of the cases, in cytoplasm in $35 \%(n=7)$, and both in nuclei and cytoplasm in $10 \%(n=2)$ of the cases.

In the group of follicular variant of PTC, positive staining was observed in $89 \%$ of the cases $(n=8 / 9)$. The staining signal of cyclin A was observed predominantly in the cytoplasm in $67 \%(n=6)$ of the cases and both in nuclei and cytoplasm in $22 \%(n=2)$.

In the group of other non-classic variants of PTC, positive staining was observed in $100 \%$ of the cases $(n=11 / 11)$. The staining signal of cyclin A was observed predominantly in the nuclei in $9.1 \%(n=1)$ of the cases, in cytoplasm in $54.5 \%(n=6)$, and both in nuclei and cytoplasm in $36.4 \%(n=4)($ Tab. 3$)$.

Cyclin B1 expression. On analyzing cyclin B1 expression, positive staining was observed in $90 \%$ of the cases $(n=36 / 40)$. Cyclin B1 immunoreactivity was observed mainly in cytoplasm in $62.5 \%(n=25 / 40)$ of cases. The staining signal of cyclin B1 was observed in the nuclei in $7.5 \%(n=3)$ of the cases, and both in nuclei and cytoplasm in $20 \%(n=8)$.

In the group of classic variant of PTC we observed positive staining in $85 \%$ of the cases $(n=17 / 20)$. The staining signal of cyclin B1 was observed predominantly in the nuclei in 10\% $(n=2)$ of the cases, in cytoplasm in $65 \%(n=13)$ of the cases and both in nuclei and cytoplasm in $10 \%(n=2)$ of the cases.

In the group of follicular variant of PTC, positive staining was observed in $89 \%$ of the cases $(n=8 / 9)$. The staining signal of cyclin B1 was observed predominantly in the cytoplasm in $67 \%(n=6)$ of the cases and both in nuclei and cytoplasm in $22 \%(n=2)$.

In the group of other non-classic variants of PTC, positive staining was observed in $100 \%$ of the cases $(n=11 / 11)$. The staining signal of cyclin B1was observed predominantly in the nuclei in $9.1 \%$ (1) of the cases, in cytoplasm in $54.5 \%(n=6)$, and both in nuclei and cytoplasm in $36.4 \%(n=4)$ (Tab. 4).

Table 3. Cyclin A staining index values for particular variants of PTC

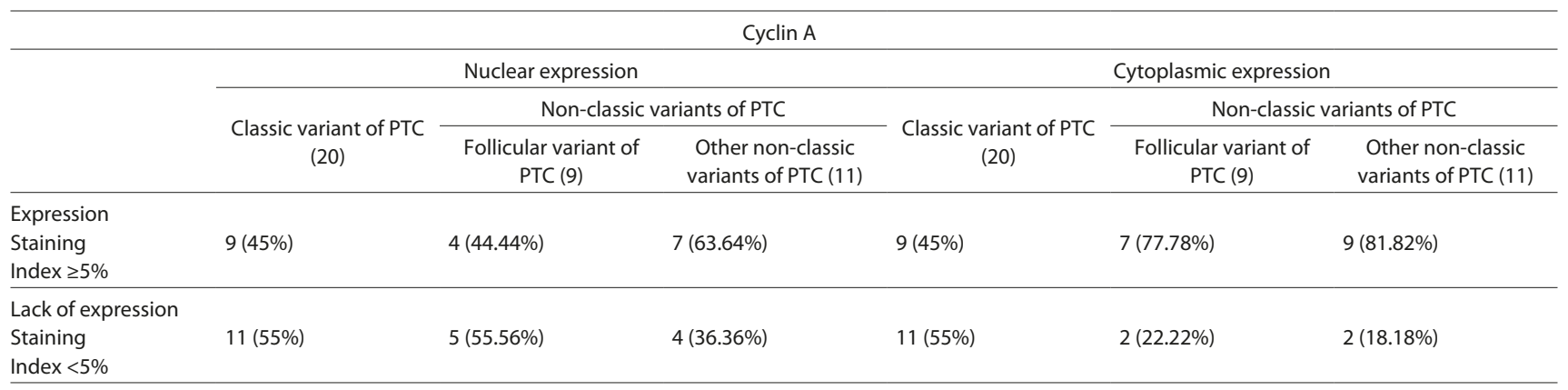

Table 4. Cyclin B1 staining index values for particular variants of PTC

\begin{tabular}{|c|c|c|c|c|c|c|}
\hline \multicolumn{7}{|c|}{ Cyclin B1 } \\
\hline & \multicolumn{3}{|c|}{ Nuclear expression } & \multicolumn{3}{|c|}{ Cytoplasmic expression } \\
\hline & $\begin{array}{l}\text { Classic variant of PTC } \\
\text { (20) }\end{array}$ & \multicolumn{2}{|c|}{ Non-classic variants of PTC } & $\begin{array}{l}\text { Classic variant of PTC } \\
\qquad(20)\end{array}$ & \multicolumn{2}{|c|}{ Non-classic variants of PTC } \\
\hline Expression & & & & & & \\
\hline $\begin{array}{l}\text { Staining } \\
\text { Index } \geq 5 \%\end{array}$ & $4(20 \%)$ & $2(22.22 \%)$ & $5(45.45 \%)$ & $15(75 \%)$ & $8(88.89 \%)$ & $10(90.92 \%)$ \\
\hline Lack of expre & & & & & & \\
\hline $\begin{array}{l}\text { Staining } \\
\text { Index <5\% }\end{array}$ & $16(80 \%)$ & $7(77.78 \%)$ & $6(54.55 \%)$ & $5(25 \%)$ & $1(11.11 \%)$ & 1 (9.09\%) \\
\hline
\end{tabular}


A statistically significant difference in total (cytoplasmic and nuclear) expression of cyclins among particular variants of PTC ( $\mathrm{p}=0.0034)$ was documented. In the group of other non-classic variants of PTC, the total expression of cyclins was clearly higher, and in the group of follicular variant of PTC it was of medium intensity, while in the group of classic variant of PTC the total expression was the weakest (Fig. 1).

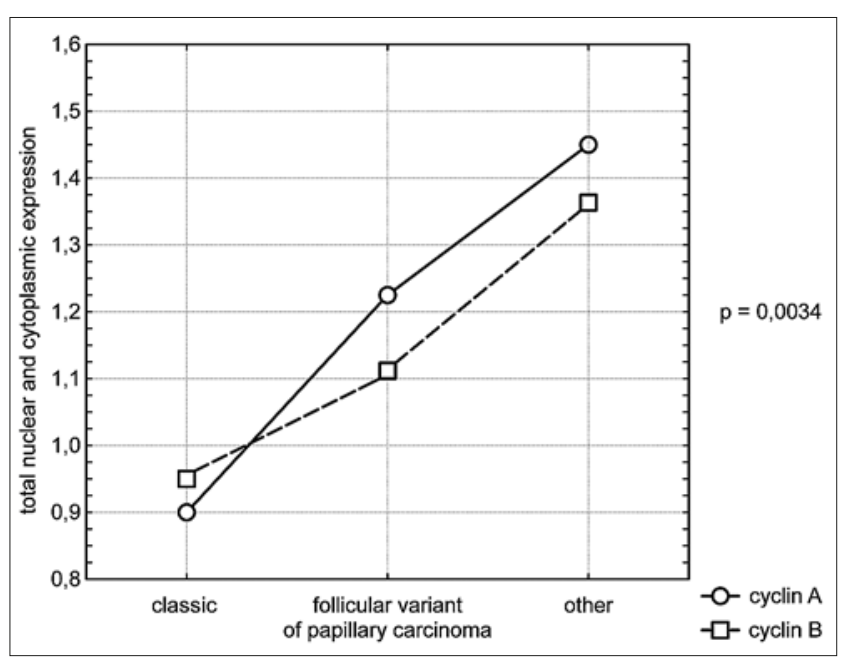

Figure 1. Total nuclear and cytoplasmic expression for particular variants of PTC

A statistically significant difference was observed in the cytoplasmic expression of cyclins among particular variants of PTC $(p=0.0386)$. In the group of other non-classic variants of PTC, the cytoplasmic expression of cyclins was clearly higher, and in the group of follicular variant of PTC it was of medium intensity, while in the group of classic variant of PTC the cytoplasmic expression was the weakest (Fig. 2).

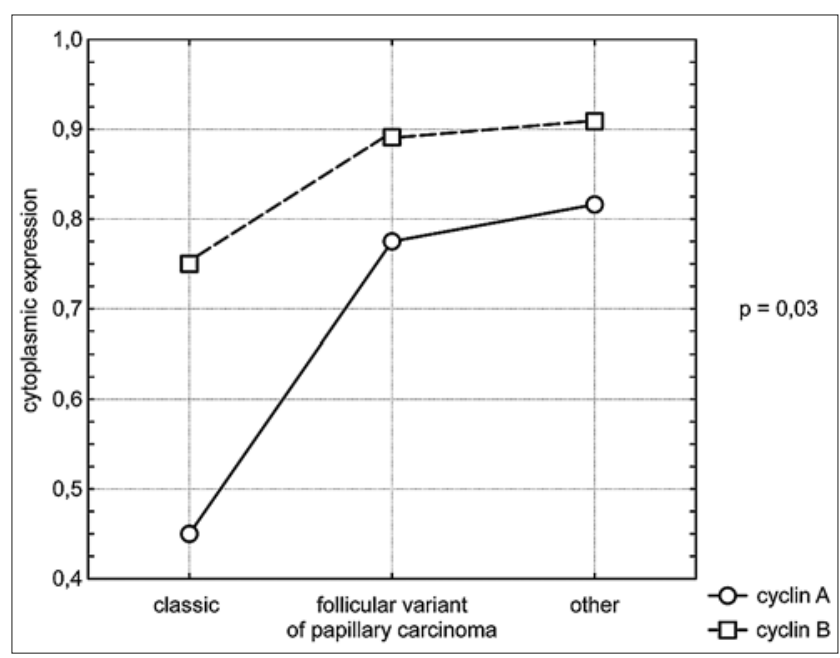

Figure 2. Cytoplasmic expression of cyclin A and B1 for particular variants of PTC

A statistically significant difference was found in the expression of cyclins A and B1 between classic and nonclassic variant of PTC $(p=0.0099)$. The expression in the classic variant of PTC was weaker. The difference reached the values of significance between the cytoplasmic and nuclear expression in the group of classic and non-classic variant of PTC $(p=0.0000)$. The nuclear expression was weaker. The difference depended on the kind of cyclin $(p=0.0036)$. For cyclin A the difference was smaller (Fig. 3).

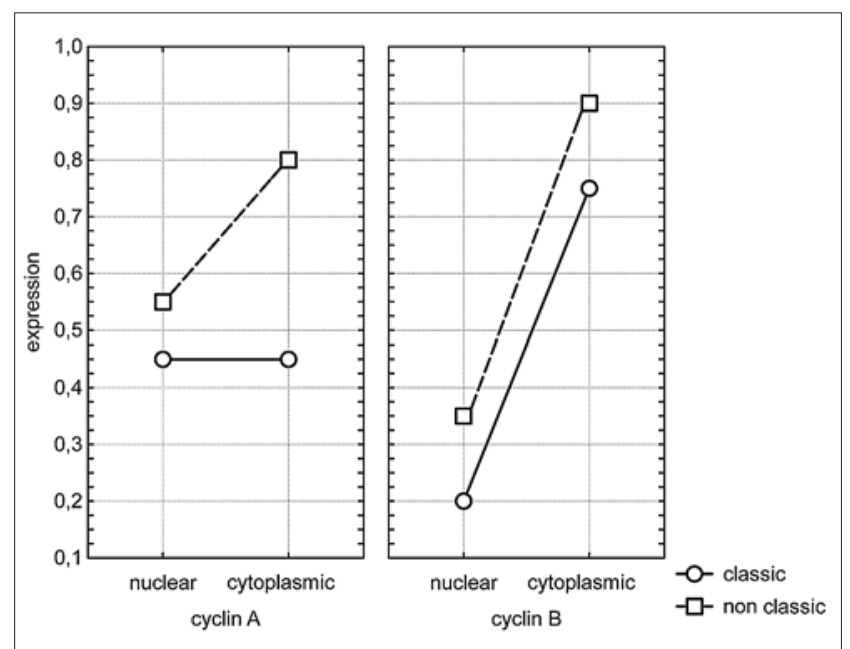

Figure 3. Nuclear and cytoplasmic expression of cyclin A and B1 for classic and non-classic variants of PTC

\section{DISCUSSION}

The pathogenesis of malignant transformation in the thyroid gland is still poorly understood. Carcinogenesis, as a result of uncontrolled cell proliferation, may result from an increased expression of cell cycle up-regulators, such as cyclins [11].

Previous studies by the authors of the presented study suggested that an increased expression of cyclin D, Pin 1 (peptidyl-prolyl cis/trans isomerase, which also plays a critical role in cell restriction points - particular in G1/S phase) and cyclin E, could play an important role in PTC carcinogenesis $[11,12,13,14]$. However, the role of other cell cycle regulatory proteins in PTC remains largely undefined.

In the present study, an attempt was made to analyze cell cycle regulators in different variants of PTC by immunohistochemical expression of a panel of two cyclins. Cyclin A and cyclin B1 were selected because these proteins exert a very important influence on cell cycle progression, and also because their role in PTC development has been less studied.

Expression of cyclin A and B1 was assessed in a number of neoplasms in humans, among others in cancers of the breast, colon, stomach and lung. In some of them, the expression of these proteins is considered to be a prognostic factor with proven clinical utility. Breast cancer seems to be the best example, as the intense expression of cyclin A in primary tumour cells is proven to be associated with an increased risk of relapse, and poor response to treatment with tamoxifen [15]. Baldini et al. [16] suggest using the expression of cyclin $A$ as an additional factor to proceed in some patients into a group with high risk of relapse. Numerous studies have also demonstrated the usefulness of the evaluation of cyclin B1 expression as a prognostic factor for breast cancer. The expression level correlated with tumour size, presence of distant metastases and disease free survival [17].

Several studies have revealed overexpression of cyclin $\mathrm{A}$ and $\mathrm{B} 1$ in thyroid cancers, including follicular thyroid carcinoma (FTC), also its poorly differentiated form and thyroid lymphoma $[18,19,20]$. Mainly, cyclin A has been overexpressed in anaplastic thyroid carcinoma [21]. Ito et al. [21] have found that cyclin A immunoexpression was 
frequently observed in undifferentiated thyroid carcinoma, indicating that this cyclin and not cyclin B1 plays a crucial role in its development. In the immunohistochemical assessment of PTC of different sizes, Dinets et al. [22] have showed that cyclin A expression has been significantly higher in PTC of size over $2 \mathrm{~cm}$, which may indicate a role of cyclin A in thyroid carcinoma de-differentation. In thyroid lymphoma, both cyclins A and B1 play an important role in the cell cycle progression [20].

However, recent reports suggest that both cyclins A and B1 may be associated with progression of thyroid carcinoma. Nar et al. [23] have shown cyclin A and B1 overexpression in differentiated (follicular and papillary) carcinoma, but not in Hürthle cell carcinoma. The expression of both cyclins have not been shown in medullary carcinoma. Kebebew et al. [24] used multigene assays as diagnostic markers to identify differentially expressed genes in benign lesions versus malignant thyroid neoplasms. The cyclin B1 gene was up-regulated in malignant thyroid neoplasms (PTC, follicular variant of PTC, follicular thyroid carcinoma).

The results obtained in the presented study also indicate that both cyclin A and B1 are expressed in PTC, and has demonstrated statistically significant difference in cyclin A expression between different histological variants of PTC. The strongest expression was observed in tumours known to have the worst prognosis (tall-cell variant, columnarcell variant), weaker in the follicular variant of PTC, and poor expression in the most promising classic variant of PTC. The overexpression of cyclins observed in other than the classic variant of PTC may indicate their role in tumour aggressiveness and dedifferentiation potential. These results are in agreement with recent reports by other investigators $[21,22,23]$. Ito et al. [21] demonstrated cyclin A overexpression in poorly-differentiated and undifferentiated thyroid cancers. Consistently, overexpression of both cyclin $\mathrm{A}$ and $\mathrm{B} 1$ has been observed in malignant but not in benign thyroid tumours [23].

Although expression of the studied cyclins was weak in the classic variant of PTC, it is still possible to distinguish cyclinpositive and cyclin-negative cancers within this group. As cyclin overexpression has been demonstrated to be a marker of the worst prognosis, such differentiation might serve as a useful tool in predicting the course of the diseases. Further studies confirming this hypothesis in a large group of PTC cases of the classic variant are needed.

Patients with PTC are not a homogeneous group and the disease course and prognosis depend significantly on the variant of PTC. Therefore, from a clinical point of view, it seems important to find a marker that will not only help to distinguish between well- and poorly- differentiated thyroid cancer, but also enable the differentiation of PTC variants. To the best of the authors' knowledge, this is the first report in the literature which analyzes by immunohistochemistry, the expression of cyclins A and B1 in different variants of PTC.

In the presented study, cyclin B1 was characterized by greater difference between nuclear and cytoplasmic expression than cyclin A. This fact can be explained by an active transport of cyclin B1/CDK1 complex and its accumulation in the cytoplasm during the G2 phase. Similar observations concerning the predominance of cytoplasmic over nuclear expression of cyclin B1 was made by Suzuki et al. [25] in cases of cancer of the breast, pancreas, colon and lung.

\section{CONCLUSIONS}

The results of the presented study suggest that cyclin A and $\mathrm{B} 1$ expression may have a characteristic pattern of immunostaining for particular variants of PTC. If the results obtained are confirmed in a larger group of patients, the diagnostic panel, constructed of the antibodies against these proteins, may increase the diagnostic accuracy in PTC cases.

\section{REFERENCES}

1. Brzeziańska E, Karbownik M, Migdalska-Sęk M, PastuszakLewandowska D, Włoch J, Lewiński A. Molecular analysis of the RET and NTRK1 gene rearrangements in papillary thyroid carcinoma in the Polish population. Mutat Res. 2006; 599(1-2): 26-35.

2. Cyniak-Magierska A, Brzeziańska E, Januszkiewicz-Caulier J, Jarząb $\mathrm{B}$, Lewiński A. Prevalence of $R A S$ point mutations in papillary thyroid carcinoma; a novel mutation at codon 31 of K-RAS. Exp Clin Endocrinol Diabetes. 2007; 115(9): 594-599.

3. Brzeziańska E, Pastuszak-Lewandoska D, Wojciechowska K, MigdalskaSęk M, Cyniak-Magierska A, Nawrot E, et al. Investigation of V600E BRAF mutation in papillary thyroid carcinoma in Polish population. Neuro Endocrinol Lett. 2007; 28(4): 351-359.

4. Pines J, Hunter T. Cyclins A and B1 in the human cell cycle. Ciba Found Symp. 1992; 170: 187-204.

5. Chibazakura T, Kamachi K, Ohara M, Tane S, Yoshikawa H, Roberts JM. Cyclin A promotes S-phase entry via interaction with the replication licensing factor Mcm7. Mol Cell Biol. 2011; 31(2): 248-255.

6. Ahlin C, Aaltonen K, Amini RM, Nevanlinna H, Fjallskog ML, Blomqvist C. Ki67 and cyclin A as prognostic factors in early breast cancer. What are the optimal cut-off values? Histopathology. 2007; 51(4): 491-498.

7. Murakami H, Furihata M, Ohtsuki Y, Ogoshi S. Determination of the prognostic significance of cyclin B1 overexpression in patients with esophageal squamous cell carcinoma. Virchows Arch. 1999; 434(2): $153-158$.

8. Jin YH, Park CK. Expression of cyclin B1 and cdc2 in nodal nonHodgkin's lymphoma and its prognostic implications. J Korean Med Sci. 2002; 17 (3): 322-327.

9. DeLeilis RA, Lloyd RV, Heitz PU, Eng C. Pathology and genetics of tumours of endocrine organs. WHO classification of tumours. Lyon: IARC Press; 2004.

10. Thyroid in: American Joint Committee on Cancer. AJCC Cancer Staging Manual. $7^{\text {th }}$ ed. New York, NY: Springer 2010, pp 87-96.

11. Brzeziński J, Migodziński A, Gosek A, Tazbir J, Dedecjus M. Cyclin E expression in papillary thyroid carcinoma - relations to staging. Int J Cancer. 2004; 109(1): 102-105.

12. Brzeziańska E, Cyniak-Magierska A, Sporny S, Pastuszak-Lewandoska D, Lewiński A. Assessment of cyklin D1 gene expression as a prognostic factor in benign and malignant thyroid lesions. Neuro Endocrinol Lett. 2007; 28(4):341-350.

13. Lewiński A, Brzeziańska E, Czarnecka K, Latek J, Koptas W, CyniakMagierska A. Increased expression of PIN1 gene in papillary thyroid carcinoma. Thyroid Res. 2011; 4(1): 4.

14. Brzeziński J, Migodziński A, Toczek A, Tazbir J, Dedecjus M. Patterns of cyclin E, retinoblastoma protein, and $\mathrm{p} 21^{\mathrm{Cip} 1 /}$ WAF1 immunostaining in the oncogenesis of papillary thyroid carcinoma. Clin Cancer Res. 2005; 11(3): 1037-1043.

15. Michalides R, van Tinteren H, Balkenende A, Vermorken JB, Benraadt J, Huldij J, et al. Cyclin A is a prognostic indicator in early stage breast cancer with and without tamoxifen treatment. Br J Cancer. 2002; 86(3):402-8.

16. Baldini E, Camerini A, Sgambato A, Prochilo T, Capodanno A, Pasqualetti, et al. Cyclin A and E2F1 overexpression correlate with reduced disease-free survival in node-negative breast cancer patients. Anticancer Res. 2006; 26(6B):4415-21.

17. Suzuki T, Urano T, Miki Y, Moriya T, Akahira J, Ishida T, et al. Nuclear cyclin B1 in human breast carcinoma as a potent prognostic factor. Cancer Sci. 2007; 98(5):644-51.

18. Pulcrano M, Boukheris H, Talbot M, Caillou B, Dupuy C, Virion A, et al. Poorly differentiated follicular thyroid carcinoma: prognostic factors and relevance of histological classification. Thyroid. 2007; 17(7): 639-646. 
19. Hooft L, van der Veldt AA, Hoekstra OS, Boers M, Molthoff CF, van Diest PJ. Hexokinase III, cyclin A and galectin-3 are overexpressed in malignant follicular thyroid nodules. Clin Endocrinol (Oxf). 2008; 68(2): 252-257.

20. Ito $\mathrm{Y}$, Yoshida H, Matsuzuka F, Matsuura N, Nakamura Y, Nakamine H, et al. Cdc2 expression in primary thyroid lymphoma: its relationship with biological aggressiveness and G2 cyclins. Pathol Res Pract. 2003; 199 (8): 533-538.

21. Ito Y, Yoshida H, Nakano K, Takamura Y, Kobayashi K, Yokozawa T, et al. Expression of G2-M modulators in thyroid neoplasms: correlation of cyclin A, B1 and cdc2 with differentiation. Pathol Res Pract. 2002; 198(6): 397-402.

22. Dinets A, Hulchiy M, Sofiadis A, Ghaderi M, Höög A, Larsson C, et al. Clinical, genetic, and immunohistochemical characterization of 70 Ukrainian adult cases with postChornobyl papillary thyroid carcinoma. Eur J Endocrinol. 2012; 166(6): 1049-1060.

23. Nar A, Ozen O, Tutuncu NB, Demirhan B. Cyclin A and cyclin B1 overexpression in differentiated thyroid carcinoma. Med Oncol. 2012; 29(1): 294-300.

24. Kebebew E, Peng M, Reiff E, Duh QY, Clark OH, McMillan A. Diagnostic and prognostic value of cell-cycle regulatory genes in malignant thyroid neoplasms. World J Surg. 2006; 30(5): 767-774.

25. Suzuki H, Graziano DF, McKolanis J, Finn OJ. T cell-dependent antibody responses against aberrantly expressed cyclin B1 protein in patients with cancer and premalignant disease. Clin Cancer Res. 2005;11(4):1521-6. 